\title{
Radiation oncology outpatient medication management needs and service gaps - a cross-sectional study of patients and clinicians
}

Sam Maleki ${ }^{1}$, Marliese Alexander ${ }^{1,2}$, Chen $\mathrm{Liu}^{3}$, Danny Rischin ${ }^{2,4}$, Senthil Lingaratnam ${ }^{1}$, Tsien Fua ${ }^{2}$

${ }^{1}$ Department of Pharmacy, Peter MacCallum Cancer Centre, Victoria, Australia ${ }^{2}$ Sir Peter MacCallum Department of Oncology, University of Melbourne, Melbourne, Australia

${ }^{3}$ Department of Radiation Oncology, Peter MacCallum Cancer Centre, Victoria, Australia

${ }^{4}$ Department of Medical Oncology, Peter MacCallum Cancer Centre, Victoria, Australia

Short title: A survey of radiotherapy patients and their clinicians. 


\section{ABSTRACT}

Title: Radiation oncology outpatient medication management needs and service gaps - a cross-sectional study of patients and clinicians.

Background: Patients receiving radiotherapy for the treatment of cancer can have complex medication requirements related to the management of side-effects and impaired swallowing ability. This study surveyed patients and clinicians to identify service gaps and unmet medication management needs.

Methods: Patient and clinician surveys were developed by a multidisciplinary team based on previously validated questionnaires. The patient survey focused on medication use and adherence. The clinician survey was based around a clinical case study and focused on identifying service gaps and practice variations. This survey was disseminated to radiation oncologists, pharmacists and nurses involved with the care of head and neck or lung cancer patients in Victoria.

Results: A total of 93 surveys were completed including 53 patient surveys and 40 clinician surveys. Radiotherapy patients reported high medication usage with up to $53 \%$ taking five or more medications daily. When asked the same set of questions relating to medication education requirements, patients receiving polypharmacy reported greater needs $(72 \%)$ than recognised by the surveyed multidisciplinary clinician group (58\%). They also reported a non-adherence rate of $46 \%$. In addition, further disparities were identified in clinician practices and their approach to clinical situations which may result in conflicting advice and confusion for patients.

\section{Conclusion:}

While recognising deficiencies relating to the provision of medication information, oncologists, nurses and pharmacists underestimated patient needs for medication information, education and follow-up. Findings support the rationale for integration of pharmacy services within the radiotherapy clinics to support patient care and bridge service gaps relating to medication management. 
Key words: Radiotherapy, ambulatory care, pharmacy outpatient services, medication management, medication adherence

Word count: 249 (limit <250) 


\section{Introduction}

Patients receiving radiotherapy, with or without concurrent chemotherapy, for the treatment of cancer experience significant treatment related adverse effects with highest event rates observed among those with head and neck, lung and upper gastrointestinal tract cancers. These toxicities include mucositis, dysphagia, orofacial pain, nausea, xerostomia, dental issues and weight loss (1-6) which often results in impaired or inability to swallow medications with frequent changes to medication regimens and formulations often being required. In the meantime, patients are also introduced to a diverse range of new and high-risk medications, including combinations of analgesics, in particular opioids, as well as combinations of antiemetics, aperients and other supportive medications to manage radiation or chemotherapy-induced toxicities.

Patients receiving radiotherapy and/or chemo-radiation are predominately managed in outpatient treatment settings with heavy reliance on patient's own involvement of their care and ability to appropriately manage medications to alleviate symptoms. In this setting, underutilisation of supportive care medications correlates with poorer management of pain and nausea, which when not intervened can escalate to pain crisis, resulting in treatment interruptions and discontinuations as well as increased hospital admissions $(7,8)$.

Barriers to optimal use of medications amongst cancer patients are diverse but may include complexity of medication regimens, inadequate education or communication when changing drugs, low self-efficacy and understanding resonating with poor socio-economic and health literacy levels. Adherence with medications often decreases with age and polypharmacy (9-11). In the radiotherapy setting adherence is particularly impacted by patient's inability to swallow medications (6). Finally, there are inherent risk factors for medication misadventure and dosing errors amongst the radiotherapy population, particularly 
with substantial medication changes and when medications are administered via enteral feeding tubes (12).

Multidisciplinary clinics incorporating a clinic pharmacist can be effective in improving medication safety and to optimise medication use $(6,13)$; however for these services to be effective, a comprehensive understanding of patient medication requirements is desirable. Importantly, pharmacy services should be integrated and complement the multidisciplinary team of radiation oncologists, nurses, dieticians and speech pathologists.

This survey study aimed to identify medication management needs, service gaps and variations in clinical practice as perceived by radiotherapy patients and their clinicians, including radiation oncologists, pharmacists and nurses who work in the radiotherapy environment.

\section{Method}

This study used a cross-sectional survey design with two anonymous surveys; a paper-based patient survey and an electronic clinician survey. The study was approved by the Peter MacCallum Cancer Centre ethical review board (research number LNR/17/PMCC/43) and sponsored by the Western and Central Melbourne Integrated Cancer Service (WCMICS). The study was managed by a steering committee, which included two radiation oncologists, one medical oncologist and three pharmacists.

\section{Study population}

Patients aged over 18 years who had recently finished or completed at least four weeks of radiotherapy for the treatment of head and neck cancer were invited to participate in the survey. Patients who were unable to complete the English language survey, or who had already completed the survey at a previous visit were excluded. Given chemo-radiotherapy toxicities usually manifest after approximately 40Gy of radiation, patients who had received less than 40Gy of 
radiation were also excluded. Patients were approached while attending radiotherapy clinics over a period of six week, and asked to complete the paper based survey while waiting for their review appointment. Survey return boxes were made available at the clinic and at the pharmacy counter. Patients were also provided with reply paid envelopes.

The clinician survey was aimed at radiation oncologists, nurses and pharmacists who were involved with the care of head and neck cancer or lung cancer patients and who practiced in Victoria. The clinician survey was disseminated via two methods: radiation oncologists were identified by the research team and emailed individually; nurses and pharmacists were contacted via a departmental approach generally relying on the unit managers to identify and forward to most suitable staff.

While survey responses were never linked to respondents, distribution lists were utilised to provide phone or email reminders at two and four weeks.

\section{Survey development}

Survey design was informed by a systematic literature review which explored pharmacy outpatient clinical services in oncology and radiotherapy clinics (13).

The patient survey included 27 short questions which took approximately five minutes to complete. This survey focused on the management of treatment related side-effects, perceptions and expectations of pharmacy services, and self-reported medication use and needs. The survey was developed in line with prior experience at our cancer centre $(14,15)$. Some questions were adapted from the Medication Understanding and Use Self-Efficacy (MUSE) (16) scale. Survey questions were rated on a four point Likert scale with responses of strongly agree, agree, disagree and strongly disagree alongside a corresponding numeric scoring system of 4, 3, 2 and 1 points used to identify the overall performance for each question. A free text question was included to allow any suggestions to improve outpatient clinic 
services. Basic demographic questions including age, sex, cancer diagnosis, mode of treatment delivery and time since diagnosis, were also asked to provide context to responses and inform future practice changes.

Polypharmacy, defined as taking five or more concurrent medications (10), was assessed by the baseline number of medications and the total number of medications, including the new medications prescribed as part of the cancer treatment.

Medication adherence may be defined as the act of taking a medication as well as taking the medication correctly with regards to the prescribed dose, frequency, timing and in consideration of drug or food interactions (17). We included two questions to assess adherence which asked if patient were taking their medications 'every day' and 'on time'. For the purposes of data analysis, patients who responded with disagree or strongly disagree to either questions were classified as non-adherent.

The clinician survey included 24 questions which took approximately 15 minutes to complete. The respondents were asked to answer a series of practice related questions based on a clinical case for either head and neck cancer or lung cancer depending on the area of practice. Case questions focused on identification of practice variation by asking questions relating to the clinician's and their organisation's practices, their perceived levels of risk associated with medication safety in the radiotherapy setting, as well as their perception of pharmacy services and how they thought these services could be best utilised.

Both the patient and clinician surveys were piloted in a small population of participants $(n=10)$ with feedback guiding minor redesigns.

\section{Data analysis}

This was a descriptive study and not designed to test a pre-determined hypothesis. Outcome measures were tabulated and expressed as percentages of total survey 
respondents. Data was summarised by means, medians, and ranges for continuous valued quantities and in tabular form for ordinal and categorical valued quantities. Free-text survey items were summarised and common themes reported.

Based on a minimum survey response rate of $60 \%$ (18) a survey dissemination target of 85 was selected which aimed at receiving at least 50 responses over a period of six weeks. The minimum inclusion criterion for partially completed surveys was set at $60 \%$, by consensus.

\section{Results}

A total of 93 surveys were completed including 53 patient surveys (62\% response rate) and 40 clinician surveys (57\% response rate). The majority (92\%) of surveys were satisfactorily completed. The average age of patient respondents was 65 years (range $48-85$ ), with $73 \%$ male patients, $49 \%$ receiving radiotherapy as single-modality therapy and $51 \%$ undergoing concurrent chemo-radiation. Majority of surveyed clinicians primarily treated head and neck cancer patients $(82 \%)$ and practiced in a clinic with pharmacy services access (59\%). Further demographic details are presented in Table 1A and 1B.

Polypharmacy was reported by up to $53 \%$ of respondents. Nearly half (47\%) of all patients were prescribed 5 or more new medications as part of their cancer treatment. Accordingly, higher rates of polypharmacy were observed among patients receiving chemo-radiation; $63 \%$ compared to $56 \%$ of patients who only received radiotherapy.

At first look, $74 \%$ of respondents reported to have good medication adherence; however when results were stratified by gender, treatment type and polypharmacy, significantly higher rates of non-adherence were reported, as shown in Figure 1.

Medication safety, was reported as not "adequately managed" in the radiotherapy outpatients setting by half of the radiation oncologist respondents $(n=7)$. 
Participants, irrespective of their answer to the above question, identified 'patient incorrect use of medication', in particular opioid analgesics $(n=11)$ and antiemetics ( $\mathrm{n}=9)$ and 'lack of help seeking behaviour' $(\mathrm{n}=10)$ as important barriers to medication safety.

Patient medication management needs and service gaps were explored via questions in both the patient and clinician surveys. Seventy-two percent of patients reported adequate communication and education by the clinic staff when starting new medications and $84 \%$ of patients reported receiving reminders by the clinic staff to take medications.

When asked the same set of questions relating to medication education requirements, patients receiving polypharmacy reported greater needs than recognised by the surveyed multidisciplinary clinician group. Seventy-two percent of these patients compared to $57 \%$ of clinicians cited a need for additional assistance with medications. Similarly, $75 \%$ of this patient population compared to $65 \%$ of clinicians cited a need for written education materials such as medication lists and information sheets. Approximately half of these respondents, both patients and clinicians, identified services gaps relating to provision of medication information, reminders to take medication, and follow-up of patient medication queries (Figure 2).

Clinician's approach towards the management of refractory nausea and vomiting was unanimous, with $95 \%$ enquiring about patient's medication compliance before considering dose change or new drug therapies. They also recommended patient be referred to pharmacy for education and advice (95\%). Clinicians reported wide practice variation in their approach towards pain management. Overall, the preference was to use non-opioid analgesics ( $81 \%)$, followed by topical anaesthetics (such as lignocaine gel)(62.5\%), followed by cocaine mouthwash (62.5\%) and using opioids as a last resort (68\%). However, compared 
to nurses and pharmacists, radiation oncologists had a much higher preference $(87 \%)$ to use opioids later in the treatment algorithm.

\section{Discussion}

This survey study explored medication management needs and service gaps as reported by radiotherapy outpatients as well as the needs and requirements of radiotherapy clinicians in order to provide an ideal outpatient oncology service.

Survey response rates were in line with the general benchmark of $60 \%$ that is usually expected for similarly sized health related surveys (18). Biases related to single centre patient survey dissemination are recognised however the cohort was shown to be generally representative of larger cohorts of head and neck cancer patients $(6,19)$ and prescribed treatments aligned with standard international guidelines. The responses therefore are likely to be generalizable to other cancer patient groups. To maximise survey response rates and reduce response bias, being a limiting factor of many survey studies, we designed both patient and clinician surveys to be anonymous. Unfortunately this prohibited the interpretation of non-response rates and biases. It is also difficult to elaborate on how the non-respondents characteristics may have differed from the responding participants.

Overall, patients expressed willingness to optimise their medication use and were receptive of additional educational services. Medication management needs were particularly significant amongst patients who used five or more medications. It is widely acknowledged that high medication burden, especially if newly prescribed during a course of intensive medical treatment, may potentiate cognitive decline in the elderly $(20,21)$ thus posing a great risk with our radiotherapy patient population.

Clinician responses to case studies demonstrated variation of practice relating to how they would manage a case of refractory nausea and vomiting and in what 
order they would prescribe and escalate analgesics for the management of radiation-induced mucositis. The approach towards management of mucositis was particularly heterogeneous with some physicians preferring systemic therapies and rapid escalation to opioid analgesics whilst others had a stronger emphasis on the use of topical anaesthetics. The use of cocaine mouthwash was highly variable with some oncologists not using this product at all. Differences in practice between health professionals in the same institution may lead to patient confusion regarding their medication. Further education and harmonisation between clinic staff might mitigate this risk.

Clinicians also identified some ongoing issues with medication safety in this patient population. Medication misadventures in this setting are predominantly related to barriers to oral route of drug administration. Mucositis and dysphagia are common in patients undergoing radiotherapy, with or without concurrent chemotherapy, and if left unchecked will prove a significant challenge to medication ingestion. In addition, patients using enteral feeding tubes for medication administration require intensive education to ensure medications are used as prescribed and administered with correct technique to avoid tube blockages. Clinicians looking after these patients also require education on the ever-changing medication landscape and available drug formulations. We identified that patients who use opioids and/or cocaine mouthwash have a higher risk of medication errors, particularly if an enteral feeding tube is inserted. In one reported scenario, a patient had administered large doses of slow-release oxycodone by first crushing the tablets and then administering this via a nasogastric tube, resulting in a hospital admission. In another, cocaine mouthwash was administered via an enteral feeding tube resulting in gastrointestinal upset and inadequate pain control. Clinicians also reported similar patient medication misadventures occurring at home, often when new medications are started and when polypharmacy and low health literacy are suspected. 
Whilst core functions of outpatient pharmacy clinical services, such as medication reconciliation and patient education (13), are well defined, other auxiliary functions, such as medication adherence assessments, identification of drugrelated problems or guidance over prescribing patterns, are less explored. Pharmacists are capable of providing a diverse range of services however given financial restraints, it is essential that services are appraised and tailored to meet consumer requirements. A key finding of this study is the importance of identification of polypharmacy in radiotherapy patients, particularly if concurrent chemotherapy is prescribed. We identified that these patients report very high rates of non-compliance, which understandably, is related to high medication burden. Pharmacy services therefore should aim to priorities services to accommodate for such patients.

Patients expressed the need for additional communication from the clinic on medication changes. These changes can be communicated verbally and complemented with written material such as consumer medicine information and a medication list which can be compiled by the clinic pharmacist. Visual cues are often effective for patients who require crushing of medication particularly if administering via feeding tubes. Pharmacists have a key role in assisting with medication prescribing and in bridging gaps in medication counselling between the physicians and patients.

Patient surveys also alluded to the fact that adherence can be improved via frequent verbal reminders as well as written medication prompts provided by the clinic staff. This is particularly effective with a patient follow-up appointment at a short timeframe where further patient feedback can be collected and incorporated into the medication management plan.

Consumer liaison via survey techniques is an important activity which can reveal important information about patient populations and must be carried out periodically. Ideally researchers should use pre-existing validated questionnaires 
however given our unique patient group and clinic setting we created a novel questionnaire via consultation with a diverse range of health professionals and consumers. The pilot surveys helped troubleshoot logistical and clinical issues; in the patient survey an additional question was inserted to allow stratification of data by whether or not patient had seen a clinic pharmacist.

This cross-sectional study has identified unmet needs relating to the optimal medication management among patients receiving radiotherapy. While recognising deficiencies relating to the provision of medication information, oncologists, nurses and pharmacists somewhat underestimated patient needs for medication information, education and follow-up. Findings support the rationale for integration of pharmacy services within the radiotherapy clinic to support patient care and bridge service gaps relating to medication management. 


\begin{tabular}{lcc}
\hline Patient Survey & $\begin{array}{c}\text { Response rate } \\
n=53\end{array}$ & $\begin{array}{c}\text { Non- } \\
\text { response }^{\mathbf{a}} \\
n=32\end{array}$ \\
\hline Average age $\mathbf{6 5}$ years & 12 & 41 \\
Gender & 48 & 5 \\
Male & 35 & \\
Female & 13 & 6 \\
Other & 0 & \\
Treatment type & 47 & \\
Radiotherapy single modality & 23 & \\
Chemo-radiation & 24 & 32 \\
Polypharmacy & 53 & \\
$\geq 5$ medications in total & 28 & \\
$\geq 5$ cancer related medications & 25 & \\
Survey response returns & 53 & \\
Radiotherapy Clinic box & 12 & \\
Outpatient dispensary box & 7 & \\
Replay paid envelopes & 34 & \\
\hline Table 1A: Patient survey characteristics & & \\
\hline
\end{tabular}

\begin{tabular}{lcc}
\hline Clinician Survey & $\begin{array}{c}\text { Response rate } \\
n=40\end{array}$ & $\begin{array}{l}\text { Non- } \\
\text { response }^{\mathbf{a}}\end{array}$ \\
\hline Profession & 39 & 1 \\
Radiation oncologist & 16 & 12 \\
Pharmacist & 5 & \\
Nurse Practitioner (or specialist) & 0 & \\
Nurse Coordinator & 1 & 1 \\
Nurse (other) & 18 & \\
Tumour stream ${ }^{\text {b }}$ & 39 & 1 \\
Head and neck cancer & 32 & \\
Lung cancer & 24 & \\
Radiotherapy patient load (new patient per & 39 & \\
week) $^{\text {b }}$ & & \\
$\leq 4$ & 14 & \\
\hline 5-8 & 12 & \\
$\geq 9$ & 5 & \\
\hline Unsure/other & 8 & \\
Clinic multidisciplinary constituents & 39 & \\
Pharmacy & 23 & \\
Dietetics & 33 & \\
Speech Pathology & 26 & \\
Physiotherapy & 3 & \\
\hline
\end{tabular}




\begin{tabular}{ll}
\hline Nurse practitioner (or specialist) & 10 \\
Nurse coordinator & 21 \\
Nurse (other) & 27 \\
Other profession & 11 \\
\hline
\end{tabular}

Table 1B: Clinician survey characteristics

${ }^{a}$ Due to the anonymous nature of surveys, non-response analysis for some subcategories was not possible

${ }^{\mathbf{b}}$ Some respondents reported to care for both tumour streams

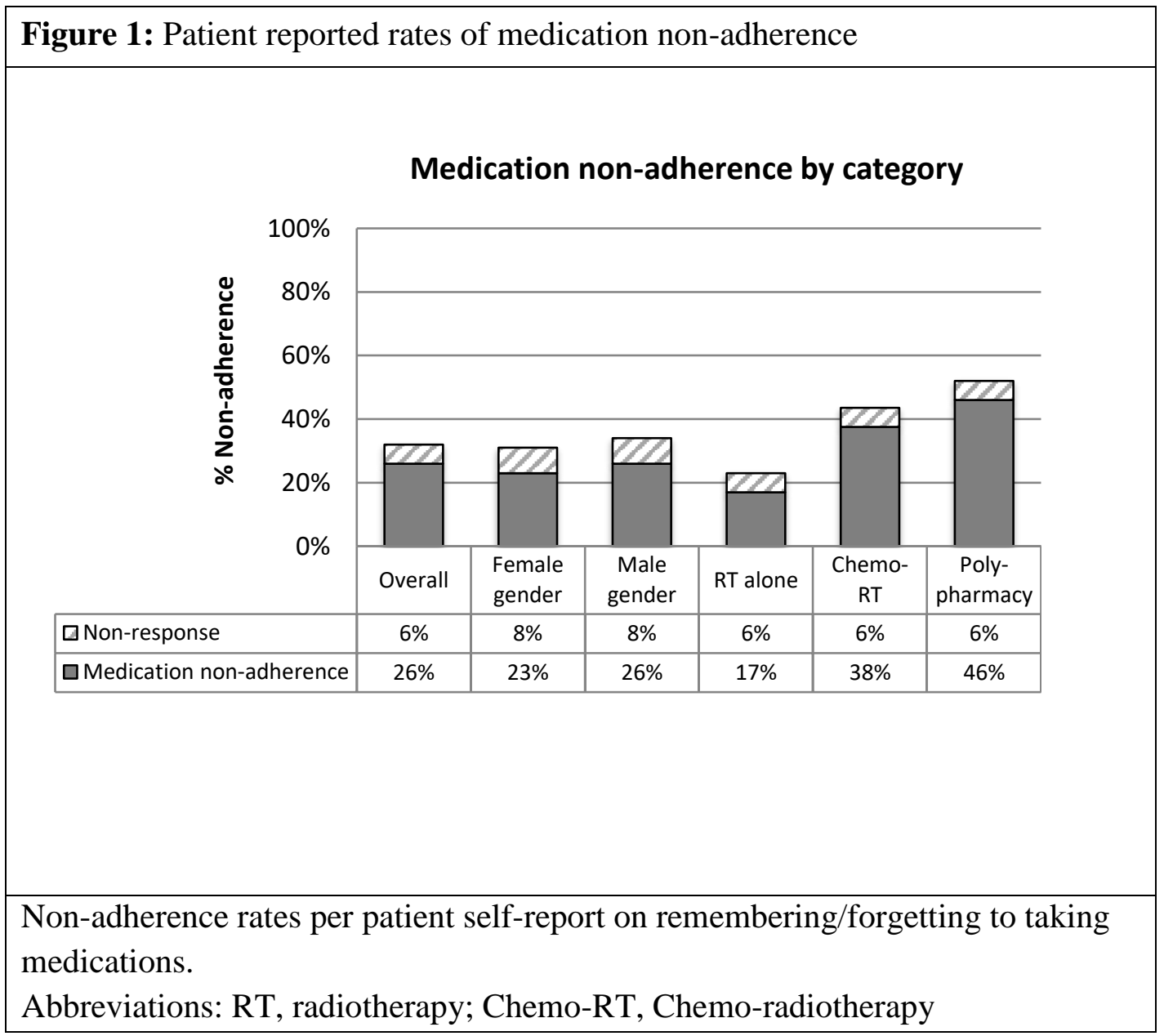




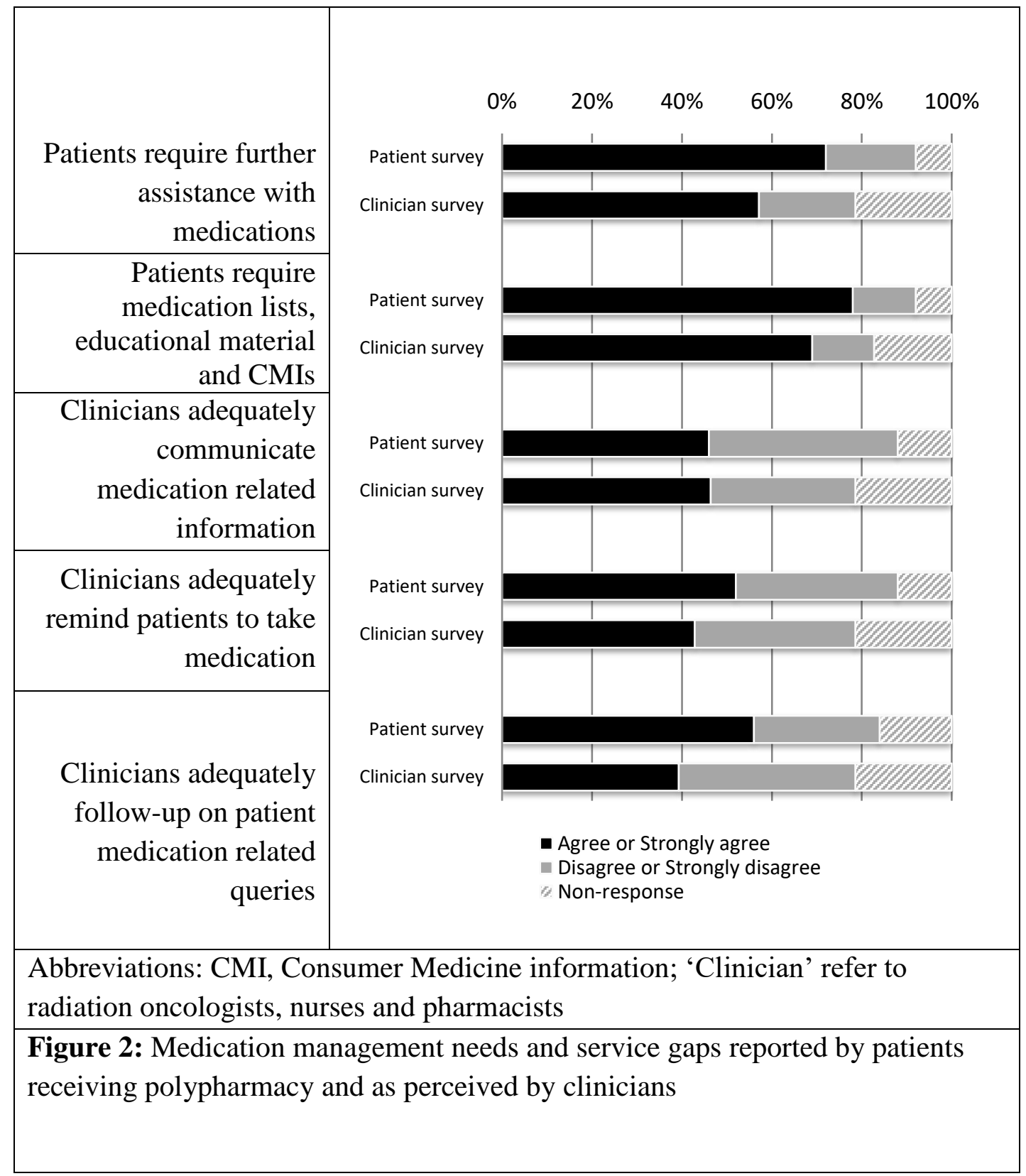




\section{References:}

1. Blanco AI, Chao KS, El Naqa I, Franklin GE, Zakarian K, Vicic M, et al. Dose-volume modeling of salivary function in patients with head-and-neck cancer receiving radiotherapy. Int J Radiat Oncol Biol Phys. 2005;62(4):1055-69.

2. Brizel DM, Albers ME, Fisher SR, Scher RL, Richtsmeier WJ, Hars V, et al. Hyperfractionated irradiation with or without concurrent chemotherapy for locally advanced head and neck cancer. The New England journal of medicine. 1998;338(25):1798-804.

3. Chao KS, Deasy JO, Markman J, Haynie J, Perez CA, Purdy JA, et al. A prospective study of salivary function sparing in patients with head-and-neck cancers receiving intensity-modulated or three-dimensional radiation therapy: initial results. Int J Radiat Oncol Biol Phys. 2001;49(4):907-16.

4. Elting LS, Cooksley CD, Chambers MS, Garden AS. Risk, outcomes, and costs of radiation-induced oral mucositis among patients with head-and-neck malignancies. Int J Radiat Oncol Biol Phys. 2007;68(4):1110-20.

5. Epstein JB, Hong C, Logan RM, Barasch A, Gordon SM, OberleeEdwards L, et al. A systematic review of orofacial pain in patients receiving cancer therapy. Supportive Care in Cancer. 2010;18(8):1023-31.

6. Scarpace SL, Brodzik FA, Mehdi S, Belgam R. Treatment of head and neck cancers: issues for clinical pharmacists. Pharmacotherapy. 2009;29(5):57892.

7. Waddle MR, Chen RC, Arastu NH, Green RL, Jackson M, Qaqish BF, et al. Unanticipated hospital admissions during or soon after radiation therapy: Incidence and predictive factors. Practical radiation oncology. 2015;5(3):e245-53. 8. De Castro G, Guindalini RS. Supportive care in head and neck oncology. Current Opinion in Oncology. 2010;22(3):221-5.

9. Lees J, Chan A. Polypharmacy in elderly patients with cancer: clinical implications and management. The Lancet Oncology. 2011;12(13):1249-57.

10. Prithviraj GK, Koroukian S, Margevicius S, Berger NA, Bagai R, Owusu C. Patient Characteristics Associated with Polypharmacy and Inappropriate Prescribing of Medications among Older Adults with Cancer. J Geriatr Oncol. 2012;3(3):228-37.

11. Wimmer BC, Cross AJ, Jokanovic N, Wiese MD, George J, Johnell K, et al. Clinical Outcomes Associated with Medication Regimen Complexity in Older People: A Systematic Review. J Am Geriatr Soc. 2017;65(4):747-53.

12. Mitchell J. Oral dosage forms that should not be crushed. Available from www.ismp.org/Tools/DoNotCrush.pdf. Accessed July 2, 2008.

13. Maleki S, Alexander M, Fua T, Liu C, Rischin D, Lingaratnam S. A systematic review of the impact of outpatient clinical pharmacy services on medication-related outcomes in patients receiving anticancer therapies. Journal of Oncology Pharmacy Practice. 2018;25(1):130-9. 
14. L M. Screening for the supportive care needs of patients with lung cancer identifies high levels of unmet need. 12th World Conference on Lung Cancer, Seoul,

Korea. 2007.

15. Walter C, Mellor JD, Rice C, Kirsa S, Ball D, Duffy M, et al. Impact of a specialist clinical cancer pharmacist at a multidisciplinary lung cancer clinic. Asia Pac J Clin Oncol. 2016;12(3):e367-74.

16. Cameron KA, Ross EL, Clayman ML, Bergeron AR, Federman AD, Bailey SC, et al. Measuring patients' self-efficacy in understanding and using prescription medication. Patient education and counseling. 2010;80(3):372-6. 17. Cramer JA, Roy A, Burrell A, Fairchild CJ, Fuldeore MJ, Ollendorf DA, et al. Medication compliance and persistence: terminology and definitions. Value in health : the journal of the International Society for Pharmacoeconomics and Outcomes Research. 2008;11(1):44-7.

18. Bennett C, Khangura S, Brehaut JC, Graham ID, Moher D, Potter BK, et al. Reporting Guidelines for Survey Research: An Analysis of Published Guidance and Reporting Practices. PLoS Medicine. 2011;8(8):e1001069. 19. American Cancer Society. Cancer facts and figures 2007. Atlanta, GA; 2007. Accessed July 31, 2007.

20. Jyrkka J, Enlund H, Lavikainen P, Sulkava R, Hartikainen S. Association of polypharmacy with nutritional status, functional ability and cognitive capacity over a three-year period in an elderly population. Pharmacoepidemiol Drug Saf. 2011;20(5):514-22.

21. Jyrkka J, Enlund H, Korhonen MJ, Sulkava R, Hartikainen S. Polypharmacy status as an indicator of mortality in an elderly population. Drugs \& aging. 2009;26(12):1039-48. 